\title{
Cervical Osteomyelitis: Unique Presentation and Fatal Outcome
}

\author{
Asma Jamil*, Ayesha Jamil, Rajesh Gulati and Liliana Crisan \\ School of Medicine Education Building, USA \\ *Corresponding author: Asma Jamil, University of California, Riverside, School of Medicine Education Building, USA
}

\begin{tabular}{|c|c|}
\hline ARTICLE INFO & ABSTRACT \\
\hline Received: 蔧 February 12, 2020 & Citation: Asma Jamil, Ayesha Jamil, Rajesh Gulati, Liliana Crisan. Cervical Osteomyelitis: \\
\hline Published: 幽 February 19, 2020 & $\begin{array}{l}\text { Unique Presentation and Fatal Outcome. Biomed J Sci \& Tech Res 25(5)-2020. BJSTR. } \\
\text { MS.ID.004257. }\end{array}$ \\
\hline
\end{tabular}

\section{Introduction}

Vertebral osteomyelitis represents $1 \%$ of the bone infection. Only 3-6\% involve the cervical spine. Its presentation can be very dramatic with rapid deterioration if immediate intervention is not done. The most common risk factors include diabetes mellitus, immunocompromised status, IV drug use, end-stage renal disease. Hematogenous spread is the most common route of infection spread to cervical spine. The involvement of cervical spine is most common at the level of C5-6 (40\%) followed by C6-7(20\%) spine with relatively uncommon involvement of C1-2 and C3-4. We are presenting a case where patient was admitted with acute hypoxic respiratory failure from diaphragmatic paralysis as a result of C1-3 vertebral osteomyelitis.

\section{Case Presentation}

A 60-year-old male, active smoker, degenerative joint disease of the cervical and lumbar spine, hypertension, admitted with the complaints of generalized weakness and worsening back pain for four days. Back pain worsened with movement. He developed acute onset paralysis of all four extremities that started 1 week before arrival. It was not cleared why he did not present earlier. His vitals on arrival were temp 87.0 F, BP 130/66 mmHg, Pulse 83, Respirate 20,02 sets $98 \%$ on room air. Physical examination revealed labored breathing and dense motor and sensory deficits in upper and lower extremities. He did not have any sensations below the T1 dermatomal level. The patient was able to communicate verbally and follow commands. Initial labs showed WBC 22.6k, Hgb 12.5, platelets 301. Electrolytes were significant for sodium 129, potassium 3.4, chloride 97, bicarb 25, BUN 8, create 0.8, anion gap 18. After arrival at the ED, patient went into cardiopulmonary arrest due to severe hypoxia and was emergently intubated. Labs and imaging studies were ordered, and broad-spectrum antibiotics were started. A chest x-ray revealed bibasal consolidation. Blood cultures ordered came back positive for methicillin-sensitive staph aureus bacteremia. Sputum culture revealed presence of candida. CT c-spine showed evidence of osteomyelitis affecting C3-4 with surrounding epidural phlegmon resulting in severe cord compression. MRI C-spine confirmed the CT scan findings. No evidence of osteomyelitis was seen at the thoracic and lumbar spine. CSF showed leukocytosis, high glucose, and protein content. Infectious disease was consulted, and patient treatment was narrowed to Nafcillin. The patient was initially weaned off from pressor but later he had episodes of worsening spinal shock manifesting in the form of hypotension and bradycardia episodes with progression to asystole. He was started on vasopressor support. Neurosurgery recommended that surgical intervention would not help given patient presentation was very late and neurological deficits recovery was very unlikely. PEG tube was placed for feeding purposes. During entire hospital course, patient was riding the ventilator and never been able to breathe above the set rate. He progressively develops worsening leukocytosis. Extensive workup revealed negative C. diff stool toxin. CT abdomen pelvis showed pneumoperitoneum with inflammatory process around duodenum. US abdomen showed ascites and 2 lit greenish fluid was tapped. Ascitic fluid revealed neutrophilic leukocytosis suggesting peritonitis and cultures were positive for candida. Antifungal medications were started. Repeat CT abdomen pelvis with contrast via PEG tube that showed 
resolution of pneumoperitoneum and no evidence of contrast extravasation. There was no evidence of visible perforation, hence patient was treated for fungal/ bacterial peritonitis. Patient repeat blood cultures remained negative on antibiotic therapy. Despite appropriate treatment, patient leukocytosis and oxygen requirements continue to increase. He developed progressive renal failure and was started on renal replacement therapy. Given patient unable to wean off from ventilator, decision was made to perform tracheostomy, however given overall poor prognosis, family decided to proceed with comfort care. Patient passed away same day after initiation of comfort measures.

\section{Discussion}

The prevalence of cervical osteomyelitis is 1 in 250000 cases. The leading causes of infections are $\mathrm{S}$ aureus, followed by Staphylococcus coagulase negative, Streptococci, Gram-negative bacilli, polymicrobial infection and fungi. According to Gupta et al, S. aureus infection and a longer time since symptoms onset to diagnosis were associated with treatment failure. Neck pain is the most presenting symptom. Other presenting symptoms are low-grade fever, chills, night sweats, fatigue, loss of appetite, neck stiffness and limited range-of-motion. The cervical osteomyelitis presentation can be very dramatic with rapid deterioration if immediate intervention is not done. If infection cause neurologic compression patients may have weakness and numbness. Compression of C3, C4 and C5 nerve that supply the diaphragm can affect breathing, making patient ventilator dependent. Physical exam may reveal tenderness and spasm or can be normal before onset of neurologic compression. Late diagnosis, neurological involvement and hospital acquisition are an independent risk factor for adverse outcome. Supportive inflammatory markers are erythrocyte sedimentation rate, C-reactive protein, and white blood cell. In most cases the diagnosis can be made using plain X-ray films and computerized tomography (CT) scan. CT scan is useful for evaluation of vertebral end plate and facet erosions from osteomyelitis.

The magnetic resonance imaging is more advantageous in evaluation of epidural compressive process of patients with neurological deficit. The causative agent can be identified by CTguided biopsy, or from blood cultures when route of infection is hematogenous spread, but the sensitivity of blood culture can be decreased by previous antibiotic therapy. Uncomplicated cases can be treated successfully with ant biotherapy for 4-8 weeks. Also, patients with associated comorbidities that make them unsuitable for surgery or with neurologic deficit persisting for more than 48 hours should benefit only by medical management. However, when possible, urgent surgical intervention is required for patients with rapidly developing neurological deficit or worsening inflammatory markers [1-5]. Our patient was unique in a way, he did not have any of the risk factors. He did not have any symptoms of neck pain or fever. His late presentation in the form of acute onset respiratory failure, quadriplegia, and ventilator-dependent status made him unsuitable candidate for surgical intervention. Unfortunately, his hospital course was complicated by persistent sepsis, peritonitis and renal failure which adversely affected his prognosis. This is the first case described in literature where cervical osteomyelitis resulted in severe cord compression and diaphragm paralysis and patient presented with respiratory arrest and quadriplegia.

\section{Learning objective}

a) Recognize diaphragm paralysis in the form of respiratory arrest as fatal outcome of cervical osteomyelitis.

b) Early recognition and timely intervention are key to survival for cervical osteomyelitis complications (Figures 1 \& 2).

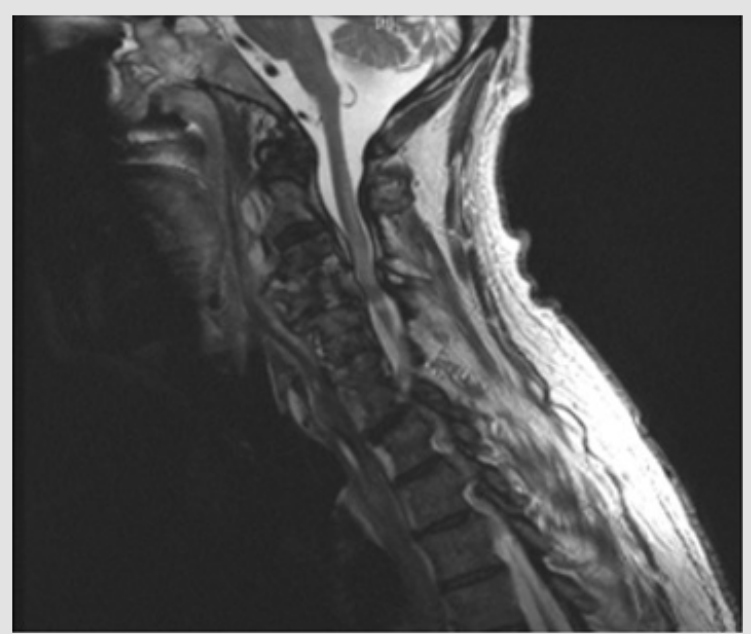

Figure 1: Sagittal T2 Flair showing hyperintense signal from $\mathrm{C} 3-\mathrm{C} 4$

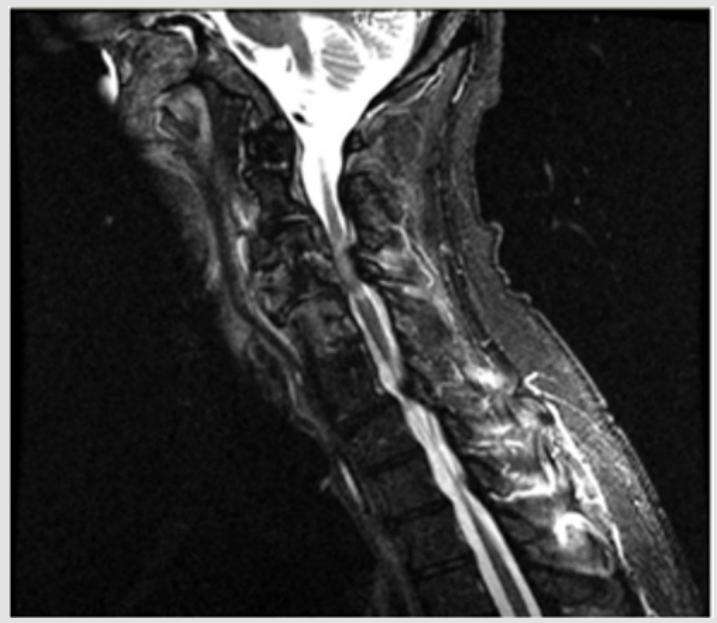

Figure 2: Sagittal T1 Flair + contrast showing bony destruction and cord compression.

\section{Disclosures/Conflict of interest}

The authors declare that there is no conflict of interest. 


\section{References}

1. Radhiana H, Win Mar Salmah J (2009) C1 and C2 vertebrae osteomyelitis: A misleading presentation leading to a fatal outcome. IMJ 8(1).

2. Schimmer RC, Jeanneret C, Nunley PD, Jeanneret B (2002) Osteomyelitis of the cervical spine: a potentially dramatic disease. J Spinal Disord Tech 15(2): 110-117.

3. Khalid Al-Hourani, Rami Al-Aref, Addisu Mesfin (2016) Upper Cervical Epidural Abscess in Clinical Practice: Diagnosis and Management. Global Spine J 6(4): 383-393.

\section{ISSN: 2574-1241}

DOI: $10.26717 /$ BJSTR.2020.25.004257

Asma Jamil. Biomed J Sci \& Tech Res

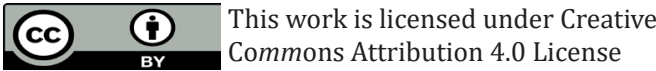

Submission Link: https://biomedres.us/submit-manuscript.php
4. Gupta A, Kowalski TJ, Osmon DR, Enzler M, Steckelberg JM, et al. (2014) Long-term outcome of pyogenic vertebral osteomyelitis: a cohort study of 260 patients. Open Forum Infect Dis 1(3): ofu 107.

5. Barnes, B, Alexander JT, Branch CL (2004) Cervical osteomyelitis: a brief review, Neurosurgical Focus FOC 17(6): 1-3.

$\begin{array}{ll}\text { BIOMEDICAL } & \text { Assets of Publishing with us } \\ \text { RESEARCHES } & \text { Global archiving of articles } \\ \text { - Immediate, unrestricted online access }\end{array}$

